\title{
Green Supply Chain Management: uma análise da produção científica recente (2001-2012)
}

\author{
Simone Sehnem ${ }^{\text {a*}}$, Charbel José Chiappetta Jabbourb ${ }^{\mathrm{b}}$, Adriana Marques Rossetto ${ }^{\mathrm{c}}$, \\ Lucila Maria de Souza Campos ${ }^{\mathrm{d}}$, Aléssio Bessa Sarquis ${ }^{\mathrm{e}}$ \\ a*simonesehnem_adm@yahoo.com.br, UNOESC e UNISUL, Brasil \\ bcharbel@usp.br, UNESP, Brasil \\ camarquesrossetto@gmail.com, UFSC, Brasil \\ lucila@deps.ufsc.br, UFSC, Brasil \\ ealessio.sarquis@unisul.br, UNISUL, Brasil
}

\section{Resumo}

0 objetivo deste estudo foi analisar a produção científica internacional na área de Green Supply Chain Management constante de produções da base de dados Business Source Complete (EBSCO Host) no período de 2001 a 2012. Pôde-se verificar a estrutura de cooperação entre autores e instituições, os autores entrantes, as categorias de produção e continuidade, a regularidade de publicação e a distribuição das publicações ao longo do tempo. Noventa artigos compuseram a amostra e os resultados evidenciaram que o número de autores que publicam é reduzido, concentrados na categoria one-timers, com 68,90\%. A maior produção foi 10 artigos por autor e o periódico mais prolífico foi o Journal of Cleaner Production, com 12 artigos publicados sobre o assunto. A Clark University (USA) destaca-se com 12 autores filiados. Conclui-se que a temática teve uma ascensão expressiva nos últimos anos.

Palavras-chave

Green Supply Chain Management. Gestão verde da cadeia de suprimentos. Bibliometria. Categorias de produção e continuidade.

\section{Introdução}

A eficácia de uma cadeia de suprimentos tradicional é mensurada com base em seu custo total e em sua rentabilidade, ignorado-se, geralmente, o impacto de sua operação sobre o meio ambiente. Entretanto, esse fenômeno está mudando rapidamente, pois os problemas ambientais decorrentes do aquecimento global e das degradações ambientais estão afetando as condições de vida da população do mundo mais severamente (Wang \& Gupta, 2011). Governos estão impondo aos fabricantes rígidas normas ambientais e diretrizes para lidarem com esses problemas. $\mathrm{Na}$ China, destacam-se novas legislações ambientais, como a Política para Economia Circular e, no Brasil, a Nova Política Nacional de Resíduos Sólidos (Lei $n^{\circ}$ 12.305, de 2 de Agosto de 2010) (Brasil, 2010). Além disso, mais conscientes das questões ambientais, os consumidores exigem produtos ecologicamente corretos, levando os fabricantes a integrarem práticas de negócios ecológicas no design do produto (o eco-design), na gestão de materiais, nos processos de fabricação, na logística, bem como em alternativas de reprocessamento dos produtos. Conforme argumentam Lai \& Wong (2012), essa crescente pressão institucional está fazendo avançar a adoção de perspectivas ambientalmente pró-ativas em temas relacionados à cadeia de suprimentos.

Nesse processo de integração de práticas e ferramentas de minimização do impacto ambiental e de tornar os produtos ecologicamente mais corretos são empregadas várias metodologias para quantificar o impacto ambiental na cadeia de fornecimento e identificar oportunidades para implantar melhorias. Dentre essas abordagens, destaca-se a Green Supply Chain Management. Wang \& Gupta (2011) consideram essa metodologia fundamental para o êxito da integração dos princípios verdes em cadeias de fornecimento. 
Nesse contexto, o triple bottom line passa a ser uma perspectiva observada pelas organizações em sua gestão. A ideia do paradigma triple bottom line é que uma corporação será bem-sucedida não apenas se tiver bom desempenho econômico-financeiro mas também pela sua função social, ética e pelo desempenho ambiental. Uma expressão muito aplicada nesse âmbito é responsabilidade social empresarial, que deve ser expressa na missão da empresa e na sua declaração dos valores. Igualmente, conforme Govindan et al. (2012), as organizações percebem que cada vez mais suas ações em compras e em gestão da cadeia de suprimentos afetam a reputação e o sucesso no longo prazo.

A seleção dos fornecedores também desempenha um papel importante na gestão da cadeia de fornecimento. Tradicionalmente, as empresas consideram critérios como preço, qualidade, agilidade e flexibilidade para avaliar o fornecedor. Mas, atualmente, os fatores de sustentabilidade desempenham um papel importante no sucesso de uma cadeia de fornecimento e do processo de compra (Govindan et al., 2012). 0 contexto acima mencionado diz respeito à incorporação dos aspectos ambientais na gestão da cadeia de suprimentos, um dos temas mais importantes da gestão organizacional contemporânea (Gunasekaran \& Spalanzani, 2012).

A Green Supply Chain Management tem se tornado foco de estudo de pesquisadores em diversos países. 0 trabalho pioneiro de Zhu \& Sakis, publicado no JOM é datado de 2004. Entretanto, de forma mais acentuada, a pesquisa nessa temática tem-se intensificado nos últimos três anos. Baseado nessa realidade, a pergunta que guiou esta pesquisa pode ser assim descrita: Como a produção científica na área de Green Supply Chain Management tem se configurado no tocante à inserção de pesquisadores na realização de pesquisas sobre essa temática? 0 objetivo do presente artigo foi analisar o papel desempenhado pelos autores no desenvolvimento da produção científica na área de Green Supply Chain Management. Quanto aos objetivos específicos, foram assim descritos: a) identificar a quantidade de artigos publicados segundo a data de publicação e por categoria de análise; b) classificar os autores dos artigos de acordo com as categorias de produção e as categorias de continuidade; c) avaliar o número de autores, a produtividade e as coautorias de cada categoria identificada; d) avaliar quais foram as instituições mais prolíficas; e) identificar os principais temas estudados pelos pesquisadores mapeados.

A originalidade desta pesquisa é dupla. Primeiramente, nela é abordado um tema recente, que vem recebendo significativa atenção internacional, inclusive com a organização de special issues
(Gunasekaran \& Spalanzani, 2012), mas que ainda não foi suficientemente abordado no Brasil. Não obstante, a sistematização do estado da arte aqui empreendida não encontra similar, até o momento, nos periódicos nacionais.

A seção 2 apresenta aspectos teóricos relacionados à gestão da cadeia de suprimentos verde. Na seção 3 é descrita a metodologia do estudo, apresentando-se a delimitação e o delineamento da pesquisa, as categorias e variáveis de análise, as técnicas de tabulação e a análise dos dados. Em seguida (seção 4), são apresentados e discutidos os resultados e apresentadas as implicações do estudo para a teoria e para a prática. Na seção 5 apresentam-se conclusões, limitações da pesquisa e sugestões para futuros estudos na área.

\section{Green Supply Chain Management (GSCM)}

Muduli et al. (2012) mencionam que a gestão da cadeia de suprimentos verde integra princípios de gestão ambiental com as atividades da cadeia de suprimentos a fim de melhorar ou preservar o ambiente, atendendo aos regulamentos ambientais e requisitos legais e sem sacrificar, como complementa Srivastava (2007), a qualidade, o custo, a confiabilidade, o desempenho e a eficiência no uso de energia. Além disso, Vachon \& Klassen (2006) destacam que ser verde tornou-se uma prática comum para retratar a imagem ambiental dos produtos, processos, sistemas e tecnologias, bem como é considerado o caminho mais adequado para a condução dos negócios.

0 conceito Green Supply Chain (GSC) foi proposto pela primeira vez em 1970 (Chen \& Liang, 2012). Sarkis et al., (2011) destacam que tudo começou com a logística reversa. No ano de 1990, a ênfase dos estudos na área foi de ordem conceitual. 0 conceito surgiu com a finalidade de proteger o meio ambiente por meio da criação de uma cultura de aprendizado na empresa, a qual deve ser assimilada com o apoio da sociedade (Chen \& liang, 2012). Ganhou notoriedade a partir da publicação de Zhu \& Sarkis (2004a). Entretanto, essa consciência está diretamente relacionada à renda das pessoas que, à medida que aumenta, as torna mais sensíveis e preocupadas com a deterioração ambiental (Dowell et al., 2000). Com vistas a reduzir o tempo que levaria a mudança de postura das empresas de forma voluntária, requisitos coercitivos têm-se formulados na legislação governamental na Europa, Ásia e América do Norte, para induzir a indústria a reduzir a poluição, por exemplo, ao exigir dos fabricantes procedimentos de logística reversa e reciclagem dos produtos verdes, práticas ampliadas com o advento da responsabilidade compartilhada com o produtor (Sheu \& Chen, 2011). 
Assim, os integrantes da cadeia de produção devem gerenciar de modo cooperativo, integrado e eficiente a cadeia de suprimentos. Empresas como IBM, Hewlett-Packard, Xerox e Body Shop International têm se envolvido com funções relacionadas a manufatura verde, remanufatura, marketing verde, logística verde e logística reversa. Kuo et al. (2010) afirmam que a seleção de fornecedores em GSCM é uma atividade fundamental na gestão de compras, porque a sustentabilidade ambiental de uma empresa e o seu desempenho ecológico podem ser demonstrados pelo desempenho dos fornecedores. Portanto, o fornecedor deve se comprometer ambientalmente também, para cooperar no alcance do desempenho almejado pela indústria e para o desenvolvimento de produtos verdes e de inovações sustentáveis.

No entanto, Sheu \& Talley (2011) e Sarkis et al., (2011) asseveram que as questões relacionadas à GSCM permanecem desafiadoras, exigindo maiores investigações. Citam como exemplo: o planejamento estratégico da GSCM e os modelos operacionais; configuração de redes em logística verde e estratégias de alocação de recursos; GSCM multilateral e canais de gestão de relacionamento; condução e promoção de incentivos para a sustentabilidade na GSCM; casos práticos de GSCM, problemas e soluções. Ruiz-Torrers et al. (2012) corroboram destacando que embora não exista um consenso em termos de classificação e/ou em uma taxonomia na área de Supply Chain Management (SCM), a maioria das pesquisas tem se centrado nas seguintes perspectivas: desenvolvimento de métodos e técnicas para estudar os processos de SCM; mensuração dos resultados do fornecimento de táticas e estratégias para a cadeia; alinhamento de prioridades competitivas para a cadeia de abastecimento; SCM verde, integrando alternativas ambientais responsáveis dentro da cadeia de suprimentos; resiliência da cadeia de suprimentos e cobertura operacional; metodologias explicativas com base na dinâmica do sistema e perspectivas multidisciplinares, considerando complexidade de interação e modelando holisticamente a cadeia de suprimentos e os sistemas logísticos.

Muduli et al. (2012) destacam que a GSCM concentra-se em uma redução dos impactos adversos das atividades da cadeia de abastecimento, assim como prima pela minimização do consumo de energia e materiais. Algumas filosofias industriais e práticas ambientais que a GSCM apoia são: sistemas de gestão ambiental, avaliação do ciclo de vida, ecologia industrial e simbiose, gestão dos produtos e responsabilidade na destinação final do produto estendida ao produtor (Muduli et al., 2012).

Ruiz-Torres et al. (2012) destacam que na América Latina os desafios da cadeia de suprimentos são numerosos e complexos. Existem evidências de que as cadeias de suprimentos de muitas empresas dessa região têm vulnerabilidades internas que podem se sobrepor aos seus esforços de participar do mercado global.

Azevedo et al. (2012) mencionam que as práticas verdes são classificadas em três níveis de análise na cadeia de suprimentos, quais sejam: a) práticas verdes desenvolvidas a montante associadas com interações ambientais com as empresas e seus fornecedores; b) práticas verdes implantadas por empresas no seu cotidiano, nas operações internas; c) práticas verdes desenvolvidas a jusante entre as empresas e seus parceiros. Zhu \& Sarkis (2004b), Zhu et al. (2011, 2012) salientam que os tipos de prática de GSCM existentes consistem em: gestão ambiental interna, compras verdes, colaboração com fornecedores, produção mais limpa, programas de auditoria e certificação ambiental, eco-design e recuperação de investimentos.

Portanto, estudar o conceito de Green Supply Chain Management (GSCM) aplicado no contexto organizacional torna-se um desafio para a academia, no intuito de contribuir para a geração de insights que contribuam para a melhoria da eficiência da cadeia de abastecimento.

\section{Metodologia}

Este trabalho foi desenvolvido utilizando a base de dados Business Source Complete (EBSCO Host) abrangendo o período de janeiro de 2001 a dezembro de 2012, com vistas a verificar a estrutura de cooperação entre autores e instituições, os autores entrantes e as categorias de produção e continuidade, a regularidade de publicação e a distribuição de suas publicações ao longo do tempo.

As palavras-chave de busca foram "Green Supply Chain Management" "e "gestão da cadeia de suprimentos verde". Foram mapeados 113 artigos. Desses, os resultados foram limitados a texto completo, tipo de publicação - academic journal, tipo de documento - artigo, idioma inglês e português, sendo que restaram 90 artigos científicos que compuseram a amostra analisada. Classificaram-se os autores em continuantes, transientes, entrantes, one-timers e retirantes.

A escolha artigos publicados em uma base de dados internacional foi feita devido à disponibilidade da mesma na instituição de ensino a que os pesquisadores estão vinculados. Outros estudos já foram desenvolvidos utilizando essa base, a exemplo de Coelho et al. (2009). Outra razão foi o fato de ser possível obter-se um maior número de publicações para a amostra num mesmo padrão de características e informações disponíveis, já que tal base de dados 
possui um número significativo de periódicos indexados disponiveis. Destaca-se também a sua importância no cenário acadêmico no que diz respeito à publicação de pesquisas científicas. Foram mapeados todos os artigos encontrados considerando-se os critérios pré-estabelecidos e supramencionados. Posteriormente, a amostra selecionada foi caracterizada em termos de linguagem e país de origem dos pesquisadores.

Para a análise dos dados, observaram-se o ano de publicação do artigo, o periódico no qual foram publicados, os autores e as instituições às quais eles se encontravam vinculados na ocasião da publicação. Quanto à identificação do vínculo institucional dos autores, ressalta-se que a obtenção de tal informação deu-se por meio dos dados constantes nos artigos científicos analisados.

Para realizar a classificação dos pesquisadores conforme as categorias de produção e continuidade utilizaram-se as tipologias mencionadas por Guarido Filho et al. (2009, 2010), que valeram da base teórica de Braun et al. (2001) e Gordon (2007). Essa análise resultou na classificação dos autores nas seguintes categorias: (a) entrantes; (b) transientes; (c) continuantes; (d) one-timers; e (e) retirantes. A definição e os critérios de cada categoria encontram-se na Tabela 1.

De posse dos artigos selecionados nessa primeira etapa, foi efetuada a tabulação dos dados e foram efetuadas as análises do estreitamento de laços entre pesquisadores.

Foram identificados quantitativamente o volume (absoluto e relativo) de artigos, de pesquisadores e de autorias presentes em cada ano e categoria. Os aspectos analisados foram: a) quantidade de artigos publicados por categoria e em quais periódicos; b) distribuição de pesquisadores segundo categorias de produção e de continuidade e coautorias relacionadas a cada categoria de produção e de continuidade; c) instituições mais prolíficas; d) autores e periódicos que publicaram sobre o tema Green Supply Chain Management.

\section{Análises e resultados}

Esta sessão encontra-se dividida em três etapas. A primeira apresenta o sumário de caracterização dos artigos analisados. A segunda, as estatísticas descritivas a respeito das cinco categorias de produção e continuidade. Na terceira destacam-se a análise bibliométrica dos temas empregados nos estudos publicados pelos autores da área de Green Supply Chain Management.

\subsection{Sumário da caracterização dos artigos}

A Tabela 2 apresenta a caracterização da amostra de artigos pesquisada.

A Tabela 2 permite constatar que a maioria dos artigos analisados foram escritos na língua inglesa e com predomínio de coautorias de múltiplos países. Destacaram-se as parcerias entre China e Estados Unidos, China, Estados Unidos e Hong Kong, Taiwan e Estados Unidos, Reino Unido e Estados Unidos, entre outras. Com menor representatividade observam-se os artigos publicados por Canadá, Índia, Japão, Alemanha, Suécia, entre outros. Sobretudo observa-se que os escritos foram elaborados em sua maioria por dois (32,22\%) ou três (30\%) autores.

\subsection{Categorias de produção e de continuidade}

A Tabela 3 apresenta a quantidade de artigos publicados por ano e por categoria.

Por meio da Tabela 3 é possível constatar que o número total de publicações sobre a temática GSCM tem aumentado ao longo do tempo. A importância que a GSCM tem nos negócios globais é certamente o fator chave que explica o aumento do número de

Tabela 1. Definição e critérios para classificação dos autores nas categorias de produção e continuidade.

\begin{tabular}{|c|c|c|}
\hline Categoria & Definição & Critérios para classificação \\
\hline \multirow{2}{*}{ Entrantes } & \multirow{2}{*}{$\begin{array}{l}\text { Novos pesquisadores na área, com publicações de pelo menos dois artigos nos últimos } \\
\text { três anos }\end{array}$} & $\geq 2$ artigos de 2010 a 2012 \\
\hline & & Sem publicações de 2001 a 2009 \\
\hline \multirow{3}{*}{ Transientes } & \multirow{3}{*}{$\begin{array}{l}\text { Pesquisadores permanentes na área, com publicações de dois ou mais artigos em no } \\
\text { máximo quatro anos, sendo que deve haver publicações tanto nos últimos três anos } \\
\text { quanto antes }\end{array}$} & $\geq 2$ artigos em até 4 anos \\
\hline & & $\geq 1$ artigo de 2010 a 2012 \\
\hline & & $\geq 1$ artigo de 2001 a 2009 \\
\hline \multirow{2}{*}{ Continuantes } & \multirow{2}{*}{$\begin{array}{l}\text { Pesquisadores consolidados na área, com publicação de pelo menos dois artigos em } \\
\text { cinco ou mais anos diferentes, inclusive nos três últimos anos }\end{array}$} & $\geq 2$ artigos em $\leq 5$ anos \\
\hline & & $\geq 1$ artigo de 2010 a 2012 \\
\hline One-timers & $\begin{array}{l}\text { Pesquisadores esporádicos, com publicação de apenas um artigo em todo o período de } \\
\text { análise }\end{array}$ & 1 artigo de 2001 a 2012 \\
\hline \multirow{2}{*}{ Retirantes } & \multirow{2}{*}{$\begin{array}{l}\text { Pesquisadores que estão deixando a área, com publicação de dois artigos, ao menos, } \\
\text { porém nenhuma publicação nos últimos três anos }\end{array}$} & $\geq 2$ artigos de 2001 a 2009 \\
\hline & & Sem publicações de 2010 a 2012 \\
\hline
\end{tabular}

Fonte: adaptada de Walter \& Bach (2012, p. 6). 
publicações nessa área. Outra possível explicação para o aumento da produção científica na área é o recente lançamento de diversos special issues sobre o tema por periódicos de significativo impacto. A categoria de destaque é a de autores one-timers, que corresponde a 68,90\% (62 artigos) da amostra de artigos analisados neste estudo (90 artigos). Em seguida apresentam-se os entrantes e continuantes, cada qual com 11 artigos publicados, o que corresponde a $12,22 \%$ para cada categoria supramencionada. Tais dados evidenciam que a temática GSCM foi recentemente incorporada no círculo de interesse da academia internacional. $\mathrm{Ou}$, ainda, que os autores que publicam nesse assunto não delimitam a expressão Green Supply Chain Management ou gestão da cadeia de suprimentos verde nas palavras-chave, no título, no resumo, restringindo a sua localização por meio da base de dados EBSCO Host. Corroborando essa possibilidade, Trierweiller et al. (2013) verificaram em um levantamento da produção científica brasileira em periódicos de Engenharia de Produção que alguns autores, apesar de estudarem a gestão ambiental e, inclusive, apresentarem sua definição no corpo do artigo, não o fazem em tópicos relevantes como:

Tabela 2. Caracterização dos artigos consultados.

\begin{tabular}{llcc}
\hline & & Número & Porcentagem (\%) \\
\hline \multirow{2}{*}{ Linguagem } & lnglês & 89 & 98,89 \\
& Português & 1 & 1,11 \\
\hline \multicolumn{2}{c}{ Distribuição geográfica } \\
\cline { 2 - 2 } País & Múltiplos países & 28 & 31,11 \\
& Taiwan & 11 & 12,22 \\
& Estados Unidos & 9 & 10 \\
& China & 6 & 6,67 \\
& Reino Unido & 5 & 5,56 \\
& Brasil & 3 & 3,33 \\
& Outros & 28 & 31,11 \\
\hline \multirow{4}{*}{ Autores } & Número de autores & por artigo & \\
& Um autor & 20 & 22,22 \\
& Dois autores & 29 & 32,22 \\
& Três autores & 27 & 30 \\
& Quatro autores & 11 & 12,22 \\
& Cinco autores & 1 & 1,11 \\
& Seis autores & 2 & 2,22 \\
\hline
\end{tabular}

Fonte: elaborada pelos autores. título, resumo e palavras-chave, campos os quais costumam ser alvo de busca em uma bibliometria. Sobremaneira, GSCM é uma área que carece, em termos internacionais, de autores entrantes, continuantes e transientes. É uma lacuna de pesquisa que pode ser preenchida inclusive por pesquisadores brasileiros que tenham interesse em aprofundar seus estudos voltados à cadeia de suprimentos alinhada aos princípios da sustentabilidade.

Considerando que a maioria dos artigos possui mais de um autor, existe a possibilidade de os autores de um mesmo artigo se enquadrarem em diferentes categorias. Para fins de esclarecimento, foram adotados critérios de quantificação, a seguir explicitados:

(1) Consiste no número de autores que podem ser classificados em cada categoria sem repetição do autor, isto é, cada autor é contabilizado apenas uma vez (independentemente do número de publicações). 0 percentual é obtido em relação ao número total de autores diferentes no período (75);

(2) Consiste no número de vezes que os autores classificados em cada categoria publicaram, isto é, admite repetição do mesmo autor conforme o número de publicações suas no período;

(3) Consiste no número de artigos em que os autores classificados em cada categoria contribuíram como autores ou coautores. A obtenção do percentual ocorre em relação ao número de artigos na amostra (91);

(4) Consiste no número de anos (de 2001 a 2012) em que houve artigo(s) publicado(s) pelos autores classificados em cada categoria. Obtém-se o percentual em relação ao número total de anos no período (12).

Na sequência, a Tabela 4 procura explicitar a distribuição dos pesquisadores pautando-se nas categorias de produção e de continuidade.

A Tabela 4 permite verificar que os autores one-timers independentemente do número de publicações correspondem a 92,09\% do total de autores que publicaram no período. Esses autores podem ser ingressantes na área, configurando um processo de iniciação e "oxigenação" no campo. Em seguida encontram-se os autores entrantes,

Tabela 3. Quantidade de artigos publicados por ano e por categoria (considerando o número de publicações).

\begin{tabular}{lccccccccccccc}
\hline \multicolumn{1}{c}{ Autores } & 2001 & 2002 & 2003 & 2004 & 2005 & 2006 & 2007 & 2008 & 2009 & 2010 & 2011 & 2012 & Total \\
\hline Entrantes & - & - & - & - & - & - & & - & - & 1 & 4 & 6 & 11 \\
Continuantes & - & - & 1 & 1 & - & 1 & 3 & 2 & - & - & 2 & 1 & 11 \\
Transientes & - & - & - & - & - & - & 1 & 1 & - & - & 1 & - & 3 \\
One-timers & 1 & - & - & - & - & 1 & 2 & 5 & 6 & 10 & 13 & 24 & 62 \\
Retirantes & 1 & - & - & 1 & 1 & - & - & - & - & - & - & - & 3 \\
Total & 2 & - & 1 & 2 & 1 & 2 & 6 & 8 & 6 & 11 & 20 & 31 & 90 \\
\hline
\end{tabular}

Fonte: elaborada pelos autores. 
Tabela 4. Distribuição dos pesquisadores segundo as categorias de produção e de continuidade.

\begin{tabular}{|c|c|c|c|c|c|c|c|c|}
\hline \multirow{2}{*}{$\begin{array}{l}\text { Categorias de } \\
\text { produção e de } \\
\text { continuidade }\end{array}$} & \multicolumn{2}{|c|}{$\begin{array}{l}\text { Autores } \\
\text { independentemente do } \\
\text { número de publicações }{ }^{1}\end{array}$} & \multicolumn{2}{|c|}{$\begin{array}{l}\text { Autores considerando } \\
\text { o número de } \\
\text { publicações }^{2}\end{array}$} & \multicolumn{2}{|c|}{ Artigos $^{3}$} & \multicolumn{2}{|c|}{$\begin{array}{c}\text { Periodos } \\
\text { com produção }{ }^{4}\end{array}$} \\
\hline & $\begin{array}{c}\text { Frequência } \\
\text { absoluta }\end{array}$ & $\begin{array}{l}\text { Frequência } \\
\text { relativa (\%) }\end{array}$ & $\begin{array}{c}\text { Frequência } \\
\text { absoluta }\end{array}$ & $\begin{array}{l}\text { Frequência } \\
\text { relativa (\%) }\end{array}$ & $\begin{array}{c}\text { Frequência } \\
\text { absoluta }\end{array}$ & $\begin{array}{l}\text { Frequência } \\
\text { relativa (\%) }\end{array}$ & $\begin{array}{c}\text { Frequência } \\
\text { absoluta }\end{array}$ & $\begin{array}{l}\text { Frequência } \\
\text { relativa (\%) }\end{array}$ \\
\hline Entrantes & 6 & 3,39 & 16 & 7,37 & 11 & 12,09 & 3 anos & 25 \\
\hline Continuantes & 2 & 1,13 & 20 & 9,22 & 11 & 12,09 & 7 anos & 58,33 \\
\hline Transientes & 5 & 2,83 & 15 & 6,91 & 3 & 3,30 & 3 anos & 25 \\
\hline One-timers & 163 & 92,09 & 164 & 75,58 & 62 & 68,13 & 8 anos & 66,67 \\
\hline Retirantes & 1 & 0,56 & 2 & 0,92 & 3 & 3,30 & 3 anos & 25 \\
\hline Total & 177 & 100 & 217 & 100 & 90 & 100 & 24 anos & 200 \\
\hline
\end{tabular}

Fonte: elaborada pelos autores.

que correspondem a 3,39\% do total de autores que publicaram no período.

A tabela também permite verificar que o número de autores que publicaram é reduzido, perfazendo um total de 177 autores distintos, e que a maioria dos artigos foram redigidos por dois pesquisadores (29 artigos). Outros 27 artigos foram redigidos por parcerias de três autores. Vinte artigos foram escritos por apenas um autor; 11 artigos por quatro autores parceiros; dois artigos por cinco autores e um artigo por seis autores.

Além disso, a Tabela 4 permite concluir que 62 artigos foram redigidos por autores one-timers e 11, por autores entrantes e por igual quantidade por autores continuantes, sendo que esses últimos redigiram seus escritos no período de 2003 a 2012 e os one-timers, por sua vez, estiveram presentes continuamente ao longo do período analisado, com um gap sem publicações nos anos de 2002, 2003, 2004 e 2005. No Brasil, em específico, o tema não era difundido. Esse alto índice de fragmentação de autorias e publicações não permite a consolidação de parcerias de publicação entre pares. Um dos possíveis motivos disso é que os artigos que compuseram essa amostra podem advir de dissertações de mestrado, teses de doutorado sob orientação de autores migrados de outras áreas, como publicações resultantes de participação temporária em grupos de pesquisa, o que se reflete na transição das temáticas de interesse de pesquisa em consonância com as tendências mundiais e a difusão da informação acerca da sustentabilidade.

\subsection{Origem dos autores que publicaram na área de Green Supply Chain Management no período de 2001 a 2012}

A Tabela 5 apresenta a origem dos autores que publicaram na área de Green Supply Chain Management no período de 2001 a 2012.

Foi possivel constatar na Tabela 5 que o país que se destacou em termos de instituições de ensino às quais os autores que publicam sobre Green Supply Chain Management estão filiados são Taiwan, Estados Unidos (USA), China e Índia. São países menos expressivos nesse ranking o Reino Unido (UK), o Brasil, a Alemanha, o Canadá, a Suécia, a Espanha, a Dinamarca, a Finlândia, Portugal, Hong Kong, Nova Zelândia, Emirados Árabes, França, Malásia, Filipinas, Japão, Austrália, Itália, Suécia, Coréia do Sul, Filipinas, Espanha, Bélgica, Irã e Turquia. Isso permite evidenciar que países europeus publicam sobremaneira sobre a temática Green Supply Chain Management e que nas Américas apresenta maior representatividade a América do Norte.

\subsection{Autores e periódicos (mais prolíficos) que publicaram sobre Green Supply Chain Management}

Na sequência, a Tabela 6 apresenta o ranking dos periódicos mais prolíficos no período analisado.

No total, foram publicados 121 artigos completos no período analisado. Desses, 90 foram publicados em periódicos qualis A1 para a área de Administração, Ciências Contábeis e Turismo (atualização 2012). Dessa amostra e conforme evidencia a Tabela 4 tiveram destaque Journal of Cleaner Production e International Journal of Production Economics, com 12 e oito artigos publicados, respectivamente, sobre Green Supply Chain Management e com fatores de impacto de 2,425 e 1,988, respectivamente. Nelas, houve maior concentração de artigos publicados nos anos de 2010, 2011 e 2012. 0 que é muito interessante é que todos esses journals promoveram special issues calls for papers sobre GSCM e isso pode explicar os resultados. $\mathrm{Na}$ sequência, o periódico Transportation Research Part E, IJPR e PSBC publicaram cada um deles cinco artigos no período analisado. Em seguida, as revistas IJBIT, Industrial Marketing Management, Procedia - Social and Behavioral Sciences e Business Strategy and the Environment publicaram cada uma quatro artigos no 
Tabela 5. Instituições mais prolíficas em relação a autores entrantes.

\begin{tabular}{|c|c|c|}
\hline Países & $\begin{array}{l}\text { Frequência } \\
\text { absoluta }\end{array}$ & $\begin{array}{l}\text { Frequência } \\
\text { relativa }(\%)\end{array}$ \\
\hline Taiwan & 13 & 14,44 \\
\hline USA & 9 & 10 \\
\hline China & 7 & 7,78 \\
\hline Índia & 7 & 7,78 \\
\hline Múltiplos países (China e USA) & 6 & 6,67 \\
\hline UK & 6 & 6,67 \\
\hline $\begin{array}{l}\text { Múltiplos países } \\
\text { (China, USA, Hong Kong) }\end{array}$ & 4 & 4,44 \\
\hline Brasil & 3 & 3,33 \\
\hline Alemanha & 3 & 3,33 \\
\hline Canadá & 2 & 2,22 \\
\hline Suécia & 2 & 2,22 \\
\hline 1tália & 2 & 2,22 \\
\hline Japão & 1 & 1,11 \\
\hline Espanha & 1 & 1,11 \\
\hline Finlândia & 1 & 1,11 \\
\hline Malásia & 1 & 1,11 \\
\hline Turquia & 1 & 1,11 \\
\hline $\begin{array}{l}\text { Múltiplos países } \\
\text { (Japão e China) }\end{array}$ & 1 & 1,11 \\
\hline Múltiplos países (UK e China) & 1 & 1,11 \\
\hline $\begin{array}{l}\text { Múltiplos países } \\
\text { (Portugal e Dinamarca) }\end{array}$ & 1 & 1,11 \\
\hline $\begin{array}{l}\text { Múltiplos países } \\
\text { (Taiwan, China e Filipinas) }\end{array}$ & 1 & 1,11 \\
\hline $\begin{array}{l}\text { Múltiplos países } \\
\text { (Índia, Dinamarca e China) }\end{array}$ & 1 & 1,11 \\
\hline $\begin{array}{l}\text { Múltiplos países } \\
\text { (China, UK e Canadá) }\end{array}$ & 1 & 1,11 \\
\hline $\begin{array}{l}\text { Múltiplos países } \\
\text { (Austrália e Índia) }\end{array}$ & 1 & 1,11 \\
\hline $\begin{array}{l}\text { Múltiplos países } \\
\text { (UK, USA e Taiwan) }\end{array}$ & 1 & 1,11 \\
\hline $\begin{array}{l}\text { Múltiplos países } \\
\text { (Portugal e França) }\end{array}$ & 1 & 1,11 \\
\hline $\begin{array}{l}\text { Múltiplos países } \\
\text { (Hong Kong, UK e Nova Zelândia) }\end{array}$ & 1 & 1,11 \\
\hline $\begin{array}{l}\text { Múltiplos países } \\
\text { (Bruxelas e Bélgica) }\end{array}$ & 1 & 1,11 \\
\hline $\begin{array}{l}\text { Múltiplos países } \\
\text { (Hong Kong e USA) }\end{array}$ & 1 & 1,11 \\
\hline Múltiplos países (USA e Índia) & 1 & 1,11 \\
\hline $\begin{array}{l}\text { Múltiplos países } \\
\text { (Dinamarca, USA e lrã) }\end{array}$ & 1 & 1,11 \\
\hline $\begin{array}{l}\text { Múltiplos países } \\
\text { (UK, Taiwan e Hong Kong) }\end{array}$ & 1 & 1,11 \\
\hline $\begin{array}{l}\text { Múltiplos países (Emirados } \\
\text { Árabes Unidos e Dinamarca) }\end{array}$ & 1 & 1,11 \\
\hline $\begin{array}{l}\text { Múltiplos países } \\
\text { (Taiwan e Filipinas) }\end{array}$ & 1 & 1,11 \\
\hline Múltiplos países (USA e Canadá) & 1 & 1,11 \\
\hline $\begin{array}{l}\text { Múltiplos países } \\
\text { (Japão, USA e Austrália) }\end{array}$ & 1 & 1,11 \\
\hline $\begin{array}{l}\text { Múltiplos países } \\
\text { (Taiwan e China) }\end{array}$ & 1 & 1,11 \\
\hline $\begin{array}{l}\text { Múltiplos países } \\
\text { (Coreia do Sul e Canadá) }\end{array}$ & 1 & 1,11 \\
\hline Total & 90 & 100 \\
\hline
\end{tabular}

Fonte: elaborada pelos autores período analisado (2001-2012). Sobretudo o ano de 2012 foi referência em publicações sobre a temática analisada, com 31 artigos divulgados sobre 0 assunto GSCM. Entretanto, 14 artigos foram publicados em periódicos distintos, que não apresentaram uma concentração representativa, ou seja, publicaram um ou dois artigos por ano analisado e, por isso, foram considerados e contabilizados na categoria Outros (periódicos).

No que se refere ao número de referências consultadas para elaborar os artigos científicos publicados houve uma variação significativa. Em alguns artigos houve apenas quatro obras consultadas. Mas houve também um artigo que consultou e referenciou 227 obras distintas para elaborar a sua publicação: o trabalho de Srivastava (2007) - um estudo bibliométrico. Na média foram consultadas 56 referências para elaborar cada artigo científico. Desconsiderando-se os 28 trabalhos discrepantes, ou seja, aqueles artigos analisados que citaram poucas referências e os que referenciaram muitos materiais, constatou-se que, dos 90 artigos, 30 artigos citaram entre 40 e 60 materiais distintos para elaborar a sua publicação. E outros 25 artigos referenciaram entre 61 e 100 obras diferentes para elaborar o seu escrito. $\mathrm{E}$, ainda, sete artigos analisados referenciaram entre 108 e 227 referências distintas para elaborar a sua redação. Isso evidencia que há uma preocupação em fundamentar de forma sólida os artigos, de modo a evidenciar o estado da arte do assunto no momento em que se gera uma nova publicação.

Em seguida, a Tabela 7 apresenta as universidades consideradas mais prolíficas e às quais os autores que publicam sobre GSCM estão filiados.

A universidade mais prolífica é a Clark University, dos USA, com 12 autores filiados, seguida pelas universidade chinesas Dalian University of Technology e Hong Kong Polytechnic University, cada uma com 10 autores filiados. Tal constatação permite a realização da inferência de que a temática GSCM tem despertado o interesse de pesquisadores chineses, taiwaneses e americanos, o que pode estar associado à realidade fabril, produtiva, de recursos disponíveis nesses países e ao nível tecnológico demandado pela cadeia de suprimentos.

Quanto aos autores que elaboraram os artigos analisados: 29 (Tabela 8) foram escritos por dois autores; 27 artigos foram elaborados por três autores; 20 foram elaborados por um autor; 11 por quatro autores distintos; um artigo por cinco autores e dois artigos por seis. Totalizando as autorias, foram mapeados 177 autores distintos para os artigos que compuseram a amostra analisada. Dentre eles, destacaram-se os autores Joseph Sarkis e Qinghua Zhu, cada qual autor e/ou coautor de 10 artigos distintos. 
Tabela 6. Periódicos mais prolíficos.

\begin{tabular}{|c|c|c|c|c|c|c|c|c|c|c|c|c|c|c|}
\hline Periódicos & $\begin{array}{l}\text { Fator de } \\
\text { impacto }\end{array}$ & 2001 & 2002 & 2003 & 2004 & 2005 & 2006 & 2007 & 2008 & 2009 & 2010 & 2011 & 2012 & Total \\
\hline $\mathrm{JCP}^{1}$ & 2,425 & - & - & 1 & - & - & 1 & - & - & - & 2 & 3 & 5 & 12 \\
\hline $1 \mathrm{JPE}^{2}$ & 1,988 & - & - & - & - & - & 1 & - & - & - & - & 1 & 6 & 8 \\
\hline TRPE $^{3}$ & 1,954 & - & - & - & - & 1 & - & - & 1 & 1 & - & 2 & - & 5 \\
\hline $1 \mathrm{JPR}^{4}$ & 1,033 & - & - & - & - & - & - & 3 & - & 1 & 1 & - & - & 5 \\
\hline $\mathrm{PSBC}^{5}$ & - & - & - & - & - & - & - & - & - & - & - & 4 & 1 & 5 \\
\hline $\mathrm{BSE}^{6}$ & - & - & - & - & - & - & - & - & 1 & - & 2 & - & 1 & 4 \\
\hline $\mathrm{IJBIT}^{7}$ & & - & - & - & - & - & - & - & - & - & - & 4 & - & 4 \\
\hline $1 \mathrm{MM}^{8}$ & 1,694 & - & - & - & - & - & - & - & - & - & - & - & 4 & 4 \\
\hline $\mathrm{GMI}^{9}$ & - & - & 2 & - & 1 & - & - & - & - & - & - & - & - & 3 \\
\hline Outros ${ }^{10}$ & - & - & - & - & 1 & - & - & 3 & 6 & 4 & 6 & 6 & 14 & 40 \\
\hline Total & - & - & 2 & 1 & 2 & 1 & 2 & 6 & 8 & 6 & 11 & 20 & 31 & 90 \\
\hline
\end{tabular}

${ }^{1}$ Journal of Cleaner Production; ${ }^{2}$ International Journal of Production Economics; ${ }^{3}$ Transportation Research Part E; ${ }^{4}$ International Journal of Production Research; ${ }^{5}$ Procedia - Social and Behavioral Sciences; ${ }^{6}$ Business Strategy and the Environment; ${ }^{7}$ International Journal of Business Information and Technology; ${ }^{8}$ Industrial Marketing Management; ${ }^{9}$ Greener Managament International; ${ }^{10}$ Engloba todos os demais periódicos nos quais foram publicados 1 ou 2 artigos no período analisado; Fonte: elaborada pelos autores.

0 autor Kee-Hung Lai foi autor e/ou coautor de seis artigos e o autor Ming-Lang Tseng, de cinco artigos. Jiuh Bung Sheu auxiliou na elaboração de quatro obras distintas. Nicolle Darnall, Dun-Ji Chen, Jiu-Bïng Sheu, Kannan Govindan, Stefan Seuring e Yenming Chen também foram autores e/ou coautores de dois artigos cada um no período analisado.

\subsection{Temas estudados pelos autores mapeados}

A Tabela 8 apresenta os temas estudados pelos autores mapeados.

Constata-se a partir da Tabela 1 que os estudos mapeados acerca de Green Supply Chain Management versam sobre assuntos teóricos e teórico-empíricos. Os estudos teóricos foram elaborados por meio de meta-análise, elaboração de frameworks, revisões teóricas que se coadunam com Green Supply Chain Management e estruturação de taxonomias.

Já os estudos teórico-empíricos abordam as iniciativas e barreiras da adoção de Green Supply Chain Management (GSCM), Green Supply Chain Management associado ao desempenho e vantagem competitiva, Green Supply Chain Management como variável importante na decisão estratégica, políticas de ecologização nas estruturas da cadeia de suprimentos, técnicas estatísticas mais sofisticadas associadas a GSCM, a exemplo da lógica fuzzy e covariância, entre outras, difusão da inovação na GSCM e o relacionamento com a melhoria organizacional.

As empresas e/ou setores nos quais os estudos mapeados foram desenvolvidos consistem em: cadeia de fornos poliéster, indústria automotiva, empresas químicas, empresas públicas e privadas, pequenas e médias empresas de diferentes setores, setor energético, indústria de celulares, agroindústria canavieira, fabricantes de computadores, stakeholders agrícolas etc.

Os principais assuntos abordados podem ser sistematizados nos seguintes tópicos:

- Gestão de custos em cadeias de produção específicas;

- Impactos da GSCM na redução de custos de processos de produção;

- GSCM associado à avaliação do ciclo de vida dos produtos;

- Práticas operacionais de GSCM, desempenho e vantagem competitiva;

- Logística operacional para GSCM;

- Produção enxuta e GSCM;

- Iniciativas, barreiras e resultados da adoção de GSCM em indústrias de manufatura;

- Como fazer a gestão da cadeia de suprimentos verde;

- GSCM e inovação verde;

- Desenvolvimento da logística de produtos na perspectiva da cadeia de suprimentos verde;

- GSCM e produção sustentável: práticas e fatores determinantes;

- Logística reversa e GSCM;

- Reciclagem e GSCM;

- Responsabilidade ambiental corporativa e GSCM;

- Sustentabilidade por meio do design verde e GSCM;

- Teoria dos jogos versus riscos ambientais versus redução das emissões de carbono na cadeia de suprimentos verde;

- GSCM e o papel da confiança e da gestão em B2B (business to business) e B2C (business to consumer);

- Aquisição verde - fornecedores verdes; 
Tabela 7. Universidades mais prolíficas.

\begin{tabular}{lcc}
\hline \multicolumn{1}{c}{ Universidade } & Frequência absoluta & Frequência relativa (\%) \\
\hline Clark University, USA & 12 & 9,35 \\
Dalian University of Technology, China & 9 & 10 \\
Hong Kong Polytechnic University, China & 4 & 10 \\
Lunghwa University of Science and Technology, Taiwan & 4,44 \\
University of Southern Denmark, Denmark & 4 & 4,44 \\
De La Salle University, Philippines & 4,44 \\
Chinese Academy of Sciences, China & 4,44 \\
George Mason University, USA & 3 & 3 \\
Tamkang University, Taiwan & 3,33 \\
National Taiwan University, Taiwan & 3 \\
National Taipei University of Technology, Taiwan & 3,33 \\
Outras & 3,33 \\
Total & 3,33 \\
\hline
\end{tabular}

Fonte: elaborada pelos autores.

- Tecnologia de informação na GSCM;

- Tomada de decisão na GSCM;

- Fatores determinantes que afetam a implantação de GSCM;

- GSCM sob a perspectiva da modernização ecológica;

- Sistema de gestão ambiental e GSCM - complementaridades para a sustentabilidade;

- Norma ISO 14.001 e GSCM;

- Impactos ambientais e GSCM;

- GSCM e criação de valor para o acionista;

- Estratégia de preços para a GSCM.

Portanto, conclui-se que os estudos sobre GSCM em sua maioria estão associados às práticas para sua adoção e disseminação ao longo da cadeia de abastecimento, com ênfase muitas vezes no elo manufatura. E, sobretudo, associados ao desempenho das organizações.

\subsection{Discussão dos resultados}

Foi possível verificar, pela análise de dados, que a produção científica que versa sobre Green Supply Chain Management era pouco expressiva no período de 2001 a 2009, sendo que no ano de 2001 foram publicados dois artigos, no ano de 2003 apenas um, no ano de 2004 dois, em 2005, um, em 2006, dois, em 2007, seis, em 2008, oito e, no ano de 2009, seis artigos. Houve um aumento no número de publicações no ano de 2010: passaram a 11 artigos publicados. Em 2011 houve novo crescimento, passando os artigos publicados sobre a temática Green Supply Chain Management para 20 e, em 2012, houve maior volume de publicações, com 31 artigos científicos sobre o assunto.

Ficou evidenciado que a maioria dos artigos escritos sobre a temática GSCM foi elaborada por poucos autores, sendo eles de autoria solitária, de duplas ou trios. Ainda, evidenciou-se que há redes sociais interuniversidades (por exemplo, instituições de ensino da China-USA; China-USA-Hong Hong) que estão se estabelecendo nessa área, inclusive interpaíses.

Essa constatação vai ao encontro dos dizeres de Jabbour et al. (2008) que, ao analisarem a produção científica sobre gestão ambiental empresarial divulgada em periódicos de administração no Brasil, no período de 1996 a 2005, constataram que há baixa quantidade de trabalhos publicados e que a falta de diversidade de autoria também se revela preocupante. Porquanto, além de haver concentração da massa crítica em gestão ambiental em determinados centros de ensino e pesquisa, grande parte da produção é relativa a um restrito número de pesquisadores. Apenas cinco instituições de ensino e pesquisa são responsáveis por quase $60 \%$ da produção observada (EAESP/FGV, USP, UFRGS, UFBA e UFSC), que se mostra, no todo ou em parte, atrelada a quatro pesquisadores (José Carlos Barbieri, Luiz Felipe Machado do Nascimento, Carmem Silva Sanches e José Célio Andrade) que, tomados em conjunto, respondem por $32 \%$ das pesquisas em gestão ambiental empresarial publicadas nos periódicos examinados.

Outro ponto de destaque foram os países de origem das publicações, com destaque para Taiwan, Estados Unidos, China e Reino Unido e apresentando como ponto diferencial a publicação multipaíses. Sobremaneira, a gestão da cadeia de suprimentos verde é um assunto que demanda a incorporação de ferramentas e técnicas de melhoria contínua, de redução dos desperdícios e minimização do impacto ambiental das atividades de produção. Geralmente, é incorporada nas organizações associada à implantação de um sistema de gestão ambiental. 


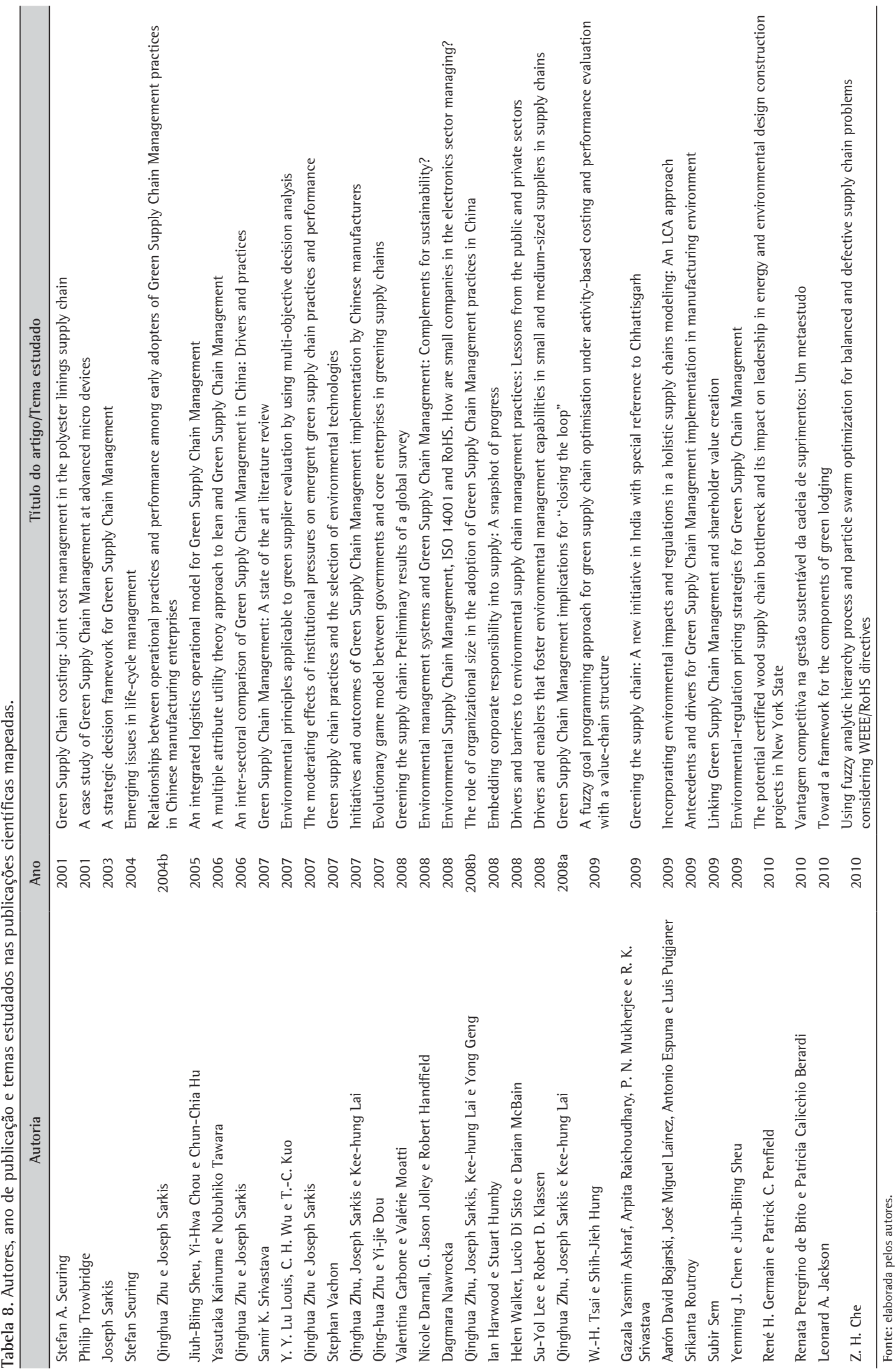




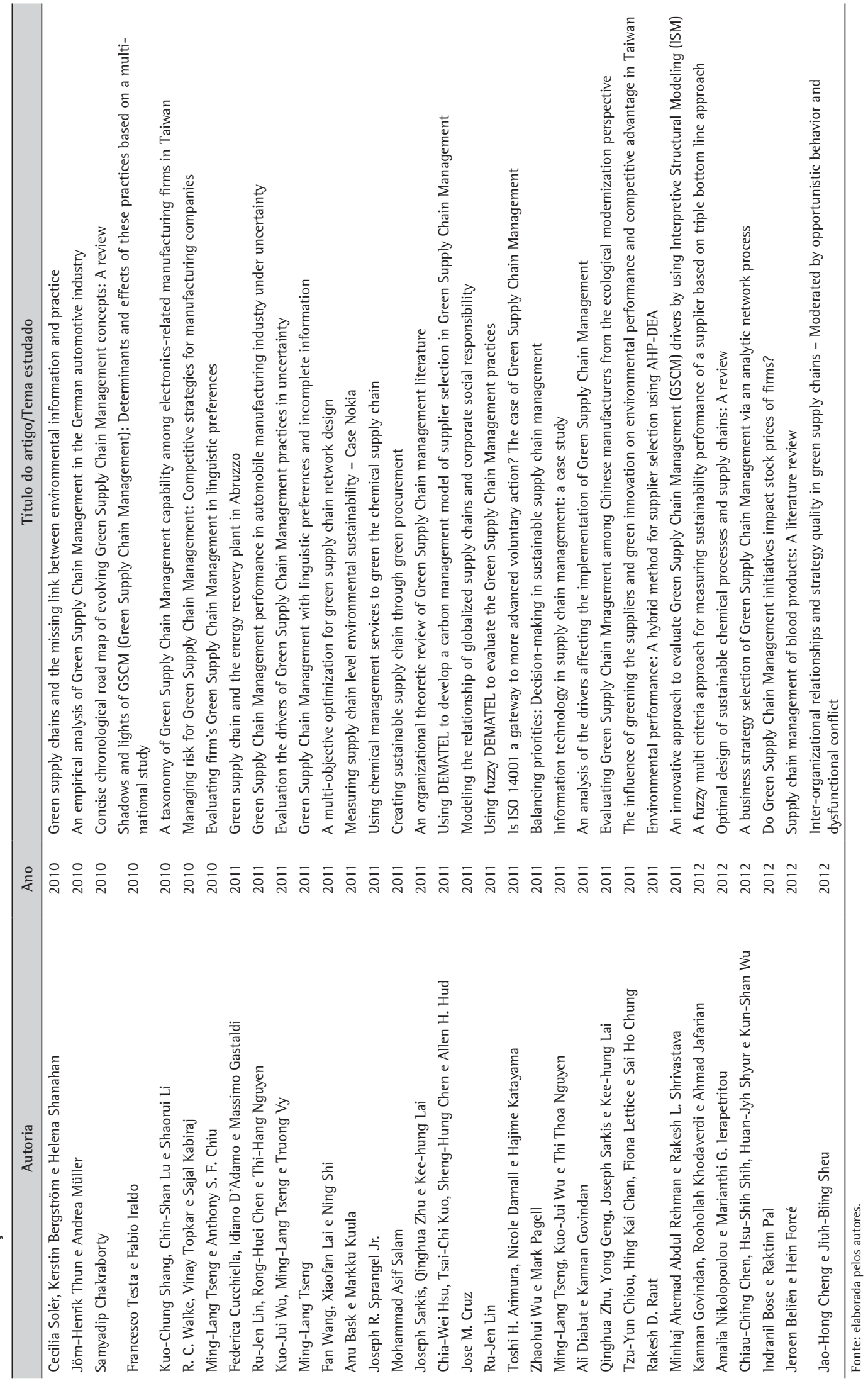




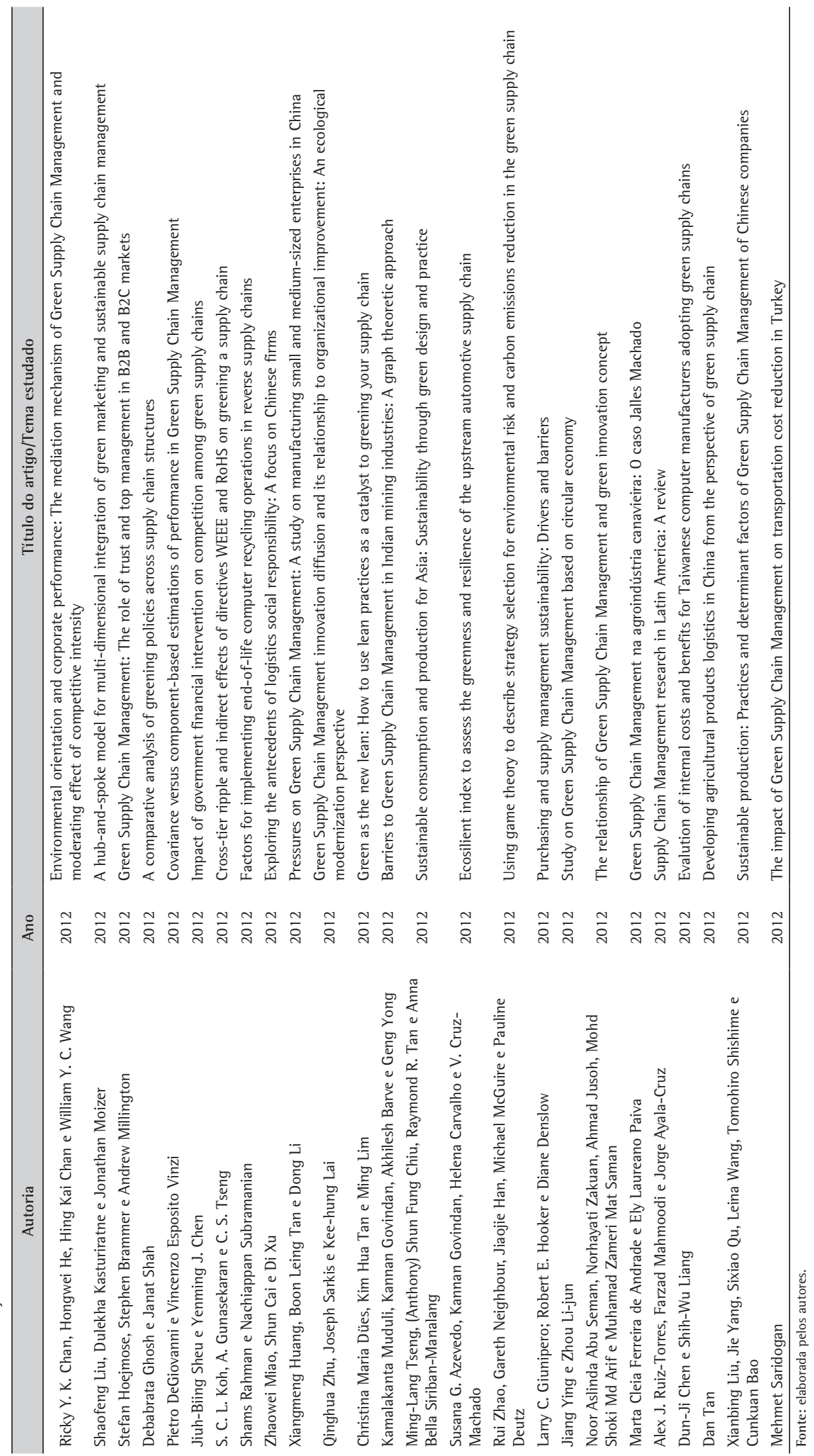


Baseado no mapeamento efetuado foi feito um diagnóstico do perfil das publicações na área de GSCM. 0 que essas informações implicam para a teoria? E para a prática da área empresarial, na gestão e na área universitária? Considera-se que o mapeamento da configuração da publicação em uma área temática em específico permite conhecer em que estágio se encontra a pesquisa naquele assunto. Isso gera oportunidades para novos estudos na área e mapeia o perfil de quem publica, das parcerias e dos vínculos.

Nesse estudo específico, além dessas informações, podem ser estabelecidas algumas análises mais gerais como, por exemplo: Por que Taiwan, Estados Unidos, China e Reino Unidos são os países que mais publicam sobre GSCM? Qual o perfil dos sistemas produtivos desses países e por que demandam sistemas de gestão e especialização para a cadeia de suprimentos? $\mathrm{Ou}$ será que a população desses países é mais consciente se comparada com a de outros países? Ou, ainda, o estágio da pesquisa nesses países é mais avançado em relação aos demais?

Esses são alguns questionamentos que surgem diante do cenário mapeado e que podem suscitar novos estudos.

\section{Conclusão}

0 objetivo do presente artigo consiste em analisar o papel desempenhado pelos autores no desenvolvimento da produção científica na área de Green Supply Chain Management. Na amostra e período analisado (2001 a 2012) foram publicados 90 artigos que envolveram 177 autores. Dos 90 artigos analisados, 11 artigos foram escritos por autores enquadrados na categoria entrantes; igual número o foi na categoria continuantes; três na categoria transientes; e o número mais representativo, 62 artigos, foi de artigos escritos por autores da categoria one-timers. Apenas 13 autores foram enquadrados em categorias diferentes da one-timers. 0 autor mais produtivo foi Joseph Sarkis, seguido por Qinghua Zhu, cada um sendo autor e/ou coautor de 10 artigos distintos. 0 autor Kee-Hung Lai foi autor e/ou coautor de seis artigos e o autor Ming-Lang Tseng, de cinco artigos. Jiuh Bung Sheu auxiliou a elaborar quatro obras distintas. Houve uma ascensão nas publicações na área no período de 2010 a 2012.

Os periódicos mais prolíficos foram o Journal of Cleaner Production e o International Journal of Production Economics, com 12 e oito artigos publicados, respectivamente, sobre Green Supply Chain Management. Na sequência, o periódico Transportation Research Part E publicou cinco artigos no período analisado. Em seguida, as revistas IJBIT, Industrial Marketing Management, Procedia - Social and Behavioral Sciences e Business Strategy and the Environment publicaram cada uma quatro artigos no período analisado (2001-2012). Deve-se notar que os periódicos mais prolíficos na área foram também aqueles que promoveram, no período de análise, calls for special issues papers que, direta ou indiretamente, exploraram a temática Green Supply Chain Management.

A universidade mais prolífica foi a Clark University, à qual 12 autores estão filiados. Dentre as americanas, ocupou posição de destaque a Dalian University of Tecnology, com nove autores filiados a ela. Entre as universidades de Taiwan, a National Taiwan University apresenta quatro filiações.

Dentre os temas pesquisados destacaram-se frameworks conceituais sobre práticas de GSCM; barreiras e motivações para adoção de GSCM; GSCM e sustentabilidade organizacional; GSCM e desempenho organizacional; logística reversa e gestão de operações no âmbito da GSCM; práticas lean e GSCM; inovação sustentável e GSCM; marketing verde e GSCM; desempenho ambiental e GSCM; e GSCM e desempenho corporativo.

Recomenda-se que estudos vindouros verifiquem a evolução do campo científico em Green Supply Chain Management na realidade dos periódicos nacionais e, além disso, ampliem a análise, tanto no âmbito brasileiro como internacional, para além dos temas aqui abordados, inclusive a abordagem metodológica dos trabalhos, os setores industriais mais retratados nos estudos sobre o tema e as tendências em GSCM. Também seria recomendável que fossem utilizadas outras bases de dados (Scopus e 1SI) em estudos semelhantes para verificar se os resultados coincidem.

\section{Referências}

Andrade, M. C. F., \& Paiva, E. L. (2012). Green supply chain management na agroindústria canavieira: o caso jalles machado. BASE - Revista de Administração e Contabilidade da Unisinos, 9(1), 2-12.

Arimura, T. H., Darnall, N., Katayama, H. (2011). ls ISO 14001 a gateway to more advanced voluntary action? The case of green supply chain management. Journal of Environmental Economics and Management, 61, 170182. http://dx.doi.org/10.1016/j.jeem.2010.11.003

Ashraf, G. Y., Raichoudhary, A., Mukherjee, P. N., \& Srivastava, R. K. (2009). Greening the supply chain: a new initiative in India with special reference to chhattisgarh. Pranjana, 12(1).

Azevedo, S. G., Govindan, K., Carvalho, H., \& Cruz-Machado, V. (2012). Ecosilient Index to assess the greenness and resilience of the upstream automotive supply chain. Journal of Cleaner Production, 1-16.

Bask, A., \& Kuula, M. (2011). Measuring Supply Chain Level Environmental Sustainability - Case Nokia. IJBIT, 3(3). 
Beliën, J., \& Forcé, H. (2012). Supply chain management of blood products: A literature review. European Journal of Operational Research, 217, 1-16. http://dx.doi. org/10.1016/j.ejor.2011.05.026

Bojarski, A. D., Laínez, J. M., Espuna, A., \& Puigjaner, L. (2009). Incorporating environmental impacts and regulations in a holistic. Computers and Chemical Engineering, 33, 1747-1759. http://dx.doi.org/10.1016/j. compchemeng.2009.04.009

Bose, 1., \& Pal, R. (2012). green supply chain management initiatives impact stock prices of firms? Decision Support Systems, 52, 624-634. http://dx.doi.org/10.1016/j. dss.2011.10.020

Brasil. (2010). Institui a Política Nacional de Resíduos Sólidos; altera a Lei no 9.605, de 12 de fevereiro de 1998. (Lei $n^{\circ} 12.305$, de 2 de agosto de 2010). Diário Oficial da República Federativa do Brasil.

Braun, T., Glänzel, W., \& Schubert, A. (2001). Publication and cooperation patterns of the authors of neuroscience journals. Scientometrics, 51(3), 499-510. http://dx.doi. org/10.1023/A:1019643002560

Brito, R. P. D., \& Berardi, P. C. (2010). Vantagem competitiva na gestão sustentável da cadeia de suprimentos: um meta-estudo. RAE, 50(2), 155-169.

Carbone, V., \& Moatti, V. (2008). Greening the Supply Chain: Preliminary Results of a Global Survey. Supply Chain Forum An International Journal, 9(2).

Chakraborty, S. (2010). Concise Chronological Road Map of Evolving Green Supply Chain Management Concepts: A Review. IUP Journal of Supply Chain Management, 7(4).

Chan, R. Y. K., He, H., Chan, H. K., \& Wang, W. Y.C. (2012). Environmental orientation and corporate performance: The mediation mecha. Industrial Marketing Management, 41, 621-630. http://dx.doi.org/10.1016/j. indmarman.2012.04.009

Che, Z. H. (2010). Using fuzzy analytic hierarchy process and particle swarm optimisation for balanced and defective supply chain problems considering WEEE/RoHS directives. International Journal of Production Research, 48(11), 33553381 . http://dx.doi.org/10.1080/00207540802702080

Chen, C. C., Shih, H. S., Shyur, H., \& Wu, K. (2012). A business strategy selection of green supply chain management via an analytic network process. Computers and Mathematics with Applications, 64, 2544-2557. http:// dx.doi.org/10.1016/j.camwa.2012.06.013

Chen, D., \& Liang, S. (2012). Evolution of internal costs and benefits for Taiwanese Computer Manufacturers Adopting Green Supply Chains. Asian Journal of Shipping and logistics, 28(1), 83-104.

Chen, Y. J., \& Sheu, J. (2009). Environmental-regulation pricing strategies for green supply chain management. Transportation Research Part E, 45, 667-677. http:// dx.doi.org/10.1016/j.tre.2009.04.010

Cheng, J., \& Sheu, J. (2012). Inter-organizational relationships and strategy quality in green supply chains - Moderated by opportunistic behavior and dysfunctional conflict. Industrial Marketing Management, 41, 563-572. http:// dx.doi.org/10.1016/j.indmarman.2012.04.003

Chiou, T. C., Chan, H. K., Lettice, F., \& Chung, S. H. (2011). The influence of greening the suppliers and green innovation on environmental performance and competitive advantage in Taiwan. Transportation Research Part E, 47, 822-836. http://dx.doi.org/10.1016/j.tre.2011.05.016
Coelho, A. L. A. L., Pavão, Y. M. P., \& Bandeira-De-Mello, R. (2009). A Produção Científica Direcionada a Visão Baseada em Recursos (RESOURCE-BASED VIEW - RBV) no Brasil e no Exterior. Revista Brasileira de Docência, Ensino e Pesquisa em Administração, 1, 177-207.

Cruz, J. M. (2011). Modeling the relationship of globalized supply chains and corporate social Responsibility. Journal of Cleaner Production, 1-13.

Cucchiella, F., D’Adamo, 1., \& Gastaldi, M. (2011). Green Supply Chain and the Energy Recovery Plant in Abruzzo. Procedia - Social and Behavioral Sciences, 25, 54-72.

Darnall, N., Jolley, G. J., \& Handfield, R. (2008). Environmental Management Systems and Green Supply Chain Management: Complements for Sustainability? Business Strategy and the Environment, 18, 30-45. http://dx.doi.org/10.1002/bse.557

Degiovanni, P., \& Vinzi, V. E. (2012). Covariance versus component-based estimations of performance in green supply chain management. International Journal of Production Economics, 135, 907-916. http://dx.doi. org/10.1016/j.jpe.2011.11.001

Diabat, A., \& Govindan, K. (2011). An analysis of the drivers affecting the implementation of green supply chain Management. Resources, Conservation and Recycling, 55, 659-667. http://dx.doi.org/10.1016/j. resconrec.2010.12.002

Dowell, G., Hart, S., \& Yeung, B. (2000). Do corporate global environmental standards create or destroy market value? Management Science, 46(6), 1059-1074. http://dx.doi. org/10.1287/mnsc.46.8.1059.12030

Dües, C. M., Tan, K. H., \& Lim, M. (2012). Green as the new Lean: how to use lean practices as a catalyst to greening your supply chain. Journal of Cleaner Production, 1-8.

Germain, R. H., \& Penfield, P. C. (2010). The Potential Certified Wood Supply Chain Bottleneck and Its Impact on Leadership in Energy and Environmental Design Construction Projects in New York State. Forest Products Journal, 60(2).

Ghosh, D., \& Shah, J. (2012). A comparative analysis of greening policies across supply chain structures. International Journal of Production Economics, 135, 568-583. http://dx.doi.org/10.1016/j.ijpe.2011.05.027

Giunipero, L. C., Hooker, R., \& Denslow, D. (2012). Purchasing and supply management sustainability: Drivers and barriers. Journal of Purchasing \& Supply Management, 18, 258-269. http://dx.doi.org/10.1016/j. pursup.2012.06.003

Gordon, A. (2007). Transient and continuant authors in a research field: the case of terrorism. Scientometrics, 72(2), 213-224. http://dx.doi.org/10.1007/s11192-0071714-Z

Govindan, K., Khodaverdi, R., \& Jafarian, A. (2012). A fuzzy multi criteria approach for measuring sustainability performance of a supplier based on triple bottom line approach. Journal of Cleaner Production, 1-10.

Guarido Filho, E. R., Machado-Da-Silva, C. L., \& Gonçalves, S. A. (2009). Institucionalização da teoria institucional nos contextos dos estudos organizacionais no Brasil. In Encontro da Associação Nacional dos Programas de PósGraduação em Administração, São Paulo.

Guarido Filho, E. R., Machado-Da-Silva, C. L., \& Rossoni, L. (2010). The Social and Intellectual Dimensions in The Construction of Scientific Knowledge: The Institutional 
Theory in Organization Studies in Brazil. BAR. Brazilian Administration Review, 7, 136-154. http://dx.doi. org/10.1590/S1807-76922010000200003

Gunasekaran, A., \& Spalanzani, A. (2012). Sustainability of manufacturing and services: Investigations for research and applications. International Journal of Production Economics, 140(1), 35-47. http://dx.doi.org/10.1016/j. ijpe.2011.05.011

Harwood, 1., \& Humby, S. (2008). Embedding corporate responsibility into supply: A snapshot of progress. European Management Journal, 26, 166-174. http:// dx.doi.org/10.1016/j.emj.2008.01.005

Hoejmose, S., Brammer, S., \& Millington, A. (2012). "Green" supply chain management: The role of trust and top management in $\mathrm{B} 2 \mathrm{~B}$ and $\mathrm{B} 2 \mathrm{C}$ markets. Industrial Marketing Management, 41, 609-620. http://dx.doi. org/10.1016/j.indmarman.2012.04.008

Hsu, C., Kuo, T., Chen, S., \& Hu, A. H. (2011). Using DEMATEL to develop a carbon management model of supplier selection in green supply chain management. Journal of Cleaner Production, 1-9.

Huang, X., Tan, B. L., \& Li, D. (2012). Pressures on Green Supply Chain Management: A Study on Manufacturing Small and Medium-Sized Enterprises in China. International Business and Management, 4(1), 76-82.

Jabbour, C. J. C., Santos, F. C. A., \& Barbieri, J. C. (2008). Gestão Ambiental Empresarial: um Levantamento da Produção Científica Brasileira Divulgada em Periódicos da Área de Administração entre 1996 e 2005. RAC - Revista de Administração Contemporânea, 12(3), 689-715.

Jackson, L. A. (2010). Toward a framework for the components of green lodging. Journal of Retail \& Leisure Property, 9(3), 211-230. http://dx.doi.org/10.1057/rlp.2010.6

Kainuma, Y., \& Tawara, N. (2006). A multiple attribute utility theory approach to lean and green supply chain management. International Journal of Production Economics, 101, 99-108. http://dx.doi.org/10.1016/j. ijpe.2005.05.010

Koh, S. C. L. K; Gunasekaran, A., \& Tseng, C. S. (2012). Crosstier ripple and indirect effects of directives WEEE and RoHS on greening a supply chain. International Journal of Production Economics, 140, 305-317. http://dx.doi. org/10.1016/j.ijpe.2011.05.008

Kuo, R. J., Wang, Y. C., \& Tien, F. C. (2010). Integration of artificial neural network and MADA methods for green supplier selection. Journal of Cleaner Production, 18, 1161-1170. http://dx.doi.org/10.1016/j. jclepro.2010.03.020

Lai, K., \& Wong, C. W. Y. (2012). Green logistics management and performance: Some empirical evidence from Chinese manufacturing exporters. Omega, 40, 267-282. http:// dx.doi.org/10.1016/j.omega.2011.07.002

Lee, S., \& Klassen, R. D. (2008). Drivers and Enablers That Foster Environmental Management Capabilities in Small- and Medium-Sized Suppliers in Supply Chains. Production and Operations Management Society, 17(6), 573-586. http://dx.doi.org/10.3401/poms.1080.0063

Lin, R. (2011). Using fuzzy DEMATEL to evaluate the green supply chain management practices. Journal of Cleaner Production, 1-8.

Lin, R.-J., Chen, R.-H., \& Nguyen, T.-H. (2011). Green supply chain management performance in automobile manufacturing industry under uncertainty Procedia - Social and Behavioral Sciences, 25, 233-245.

Liu, S., Kasturiratne, D., \& Moizer, J. (2012). A hub-andspoke model for multi-dimensional integration of green marketing and sustainable supply chain management. Industrial Marketing Management, 41, 581-588. http:// dx.doi.org/10.1016/j.indmarman.2012.04.005

Liu, X., Yang, J., Qu, S., Wang, L., Shishime, T., \& Bao, C. (2012). Sustainable Production: Practices and Determinant Factors of Green Supply Chain Management of Chinese Companies. Business Strategy and the Environment, 21(1), 1-16. http://dx.doi.org/10.1002/ bse.705

Louis, Y. Y. L., Wu, C. H., \& Kuo, T.-C. (2007). Environmental principles applicable to green supplier evaluation by using multi-objective decision analysis. International Journal of Production Research, 45(18-19), 4317-4331. http://dx.doi.org/10.1080/00207540701472694

Miao, Z., Cai, S., \& Xu, D. (2012). Exploring the antecedents of logistics social responsibility: A focus on Chinese firms. International Journal of Production Economics, 140, 18-27. http://dx.doi.org/10.1016/j.ijpe.2011.05.030

Muduli, K., Govindan, K., Barve, A., \& Yong, G. (2012). Barriers to Green Supply Chain Management in Indian Mining Industries: A Graph Theoretic Approach. Journal of Cleaner Production.

Nawrocka, D. (2008). Environmental Supply Chain Management, ISO 14001 and RoHS. How Are Small Companies in the Electronics Sector Managing? Corporate Social Responsibility and Environmental Management, 15, 349-360. http://dx.doi.org/10.1002/ csr. 176

Nikolopoulou, A., \& lerapetritou, M. G. (2012). Optimal design of sustainable chemical processes and supply chains: A review. Computers and Chemical Engineering, 44, 94-103. http://dx.doi.org/10.1016/j. compchemeng.2012.05.006

Rahman, S., \& Subramanian, N. (2012). Factors for implementing end-of-life computer recycling operations in reverse supply chains. International Journal of Production Economics, 140, 239-248. http://dx.doi. org/10.1016/j.ijpe.2011.07.019

Raut, R. D. (2011). Environmental Performance: A Hybrid Method for Supplier Selection using AHP-DEA. IJBIT, 5(1)

Rehman, M. A. B., \& Shrivastava, R. L. (2011). An innovative approach to evaluate green supply chain management (GSCM) drivers by using interpretive structural modeling (ISM). International Journal of Innovation and Technology Management, 8(2), 315-336. http://dx.doi. org/10.1142/S0219877011002453

Routroy, S. (2009). Antecedents and Drivers for Green Supply Chain Management Implementation in Manufacturing Environment. The Icfai University Journal of Supply Chain Management, 6(1), 20-35.

Ruiz-Torres, A. J., Mahmoodi, F., \& Ayala-Cruz, J. (2012). Supply Chain Management Research in Latin America: a Review. Supply Chain Forum: An International Journal, 13(1).

Salam, M. A. (2011). Creating Sustainable Supply Chain Through Green Procurement. IJBIT, 3(Sp lssue 3).

Saridogan, M. (2012). The Impact of Green Supply Chain Management on Transportation Cost Reduction in 
Turkey. International Review of Management and Marketing, 2(2), 112-121.

Sarkis, J. (2003). A strategic decision framework for green supply chain Management. Journal of Cleaner Production, 11, 397-409. http://dx.doi.org/10.1016/ S0959-6526(02)00062-8

Sarkis, J., Zhu, Q., \& Lai, K. (2011). An organizational theoretic review of green supply chain management literature. International Journal of Production Economics, 130(1), 1-15. http://dx.doi.org/10.1016/j.ijpe.2010.11.010

Sem, S. (2009). linking Green Supply Chain Management and Shareholder Value Creation. The IUP Journal of Supply Chain Management, 6(3-4).

Seman, N. A. A., Zakuan, N., Jusoh, A., Arif, M. S. M., \& Saman, M. Z. M. (2012). The relationship of green supply chain management and green innovation concept. Procedia - Social and Behavioral Sciences, 57, 453-457.

Seuring, S. A. (2001). Green Supply Chain Costing Joint Cost Management in the Polyester Linings Supply Chain (pp.71-80). GMI, Spring.

Seuring, S. (2004). Emerging Issues in Life-Cycle Management (pp. 3-8). GMl, Spring .

Shang, K; Lu, C., \& Li,S. (2010). A taxonomy of green supply chain management capability among electronics-related manufacturing firms in Taiwan. Journal of Environmental Management, 91, 1218-1226. PMid:20181423. http:// dx.doi.org/10.1016/j.jenvman.2010.01.016

Sheu, J., \& Chen, Y. J. (2012). Impact of government financial intervention on competition among green supply chains. International Journal of Production Economics, 138, 201-213. http://dx.doi.org/10.1016/j.ijpe.2012.03.024

Sheu, J., Chou, Y., \& Hu, C. (2005). An integrated logistics operational model for green-supply chain management. Transportation Research Part E, 41, 287-313. http:// dx.doi.org/10.1016/j.tre.2004.07.001

Sheu, J., \& Talley, W. (2011). Green supply chain management: trends, challenges, and solutions. Transportation Research Part E, 47(6), 791-792. http:// dx.doi.org/10.1016/j.tre.2011.05.014

Solér, C., Bergström, K., \& Shanahan, H. (2010). Green Supply Chains and the Missing link Between Environmental Information and Practice. Business Strategy and the Environment, 19, 14-25.

Sprangel Junior, J. R. 2011 (). Using Chemical Management Services to Green the Chemical Supply Chain. IJBIT, 3(3) .

Srivastava, S. K. (2007). Green supply-chain management: A state-of the-art literature review. International Journal of Management Reviews, 9(1), 53-80. http://dx.doi. org/10.1111/j.1468-2370.2007.00202.x

Tan, D. (2012). Developing Agricultural Products Logistics in China from the Perspective of Green Supply Chain. International Journal of Business and Management, 7(21). http://dx.doi.org/10.5539/ijbm.v7n21p106

Testa, F., \& 1raldo, F. (2010). Shadows and lights of GSCM (Green Supply Chain Management): determinants and effects of these practices based on a multi-national study. Journal of Cleaner Production, 18, 953-962. http://dx.doi.org/10.1016/j.jclepro.2010.03.005

Thun, J., \& Müller, A. (2010). An Empirical Analysis of Green Supply Chain Management in the German Automotive Industry. Business Strategy and the Environment, 19, 119-132.
Trierweiller, A. C., Campos, L. M. S., Carvalho, D. N., Santos, T. H. S., Bornia, A. C., \& Peixe, B. C. S. (). Gestão Ambiental: Levantamento da Produção Científica Brasileira em Periódicos de Engenharia de Produção. Produção, 2013. No prelo.

Trowbridge, P. (2001). A Case Study of Green Supply-Chain Management at Advanced Micro Devices (pp. 121-135). GMl, Autumm.

Tsai, W.-H., \& Hung, S. (2009). A fuzzy goal programming approach for green supply chain optimisation under activity-based costing and performance evaluation with a value-chain structure. International Journal of Production Research, 47(18), 4991-5017. http://dx.doi. org/10.1080/00207540801932498

Tseng, M. (2011). Green supply chain management with linguistic preferences and incomplete Information. Applied Soft Computing, 11, 4894-4903. http://dx.doi. org/10.1016/j.asoc.2011.06.010

Tseng, M., Schiu, A. S. F., Tan, R. R., \& Siriban-Manalang, A. B. (2012). Sustainable consumption and production for Asia: sustainability through green design and practice. Journal of Cleaner Production, 1-5.

Tseng, M., \& Chiu, A. S.F. (2010). Evaluating firm's green supply chain management in linguistic preferences. Journal of Cleaner Production, 1-10.

Tseng, M., Wu, K., \& Nguyen, T. T. (2011). Information technology in supply chain management: a case Study. Procedia - Social and Behavioral Sciences, 25, 257-272.

Vachon, S. (2007). Green supply chain practices and the selection of environmental Technologies. International Journal of Production Research, 45(18-19), 4357-4379. http://dx.doi.org/10.1080/00207540701440303

Vachon, S., \& Klassen, R. D. (2006). Extending green practices across the supply chain: the impact of upstream and downstream integration. International Journal of Operations and Production Management, 26(7), 795821. http://dx.doi.org/10.1108/01443570610672248

Walke, R. C., Topkar, V., \& Kabiraj, S. (2010). Managing Risk for Green Supply Chain Management: Competitive Strategies for Manufacturing Companies. Skyline Business Journal, 6(1), 1-11.

Walker, H., Sisto, L. D., \& McBain, D. (2008). Drivers and barriers to environmental supply chain management practices: Lessons from the public and private sectors. Journal of Purchasing \& Supply Management, 14, 6985. http://dx.doi.org/10.1016/j.pursup.2008.01.007

Walter, S. A., \& Bach, T. M. (2012). Inserção de pesquisadores entrantes na área de estratégia: análise das relações de autoria e temas estudados no período de 1997-2010. Revista Eletrônica de Administração (REAd). No prelo.

Wang, H., \& Gupta, S. M. (2011). Green Supply Chain Management: Product Life Cycle Approach. McGraw Hill.

Wang, F., Lai, X., \& Shi, N. (2011). A multi-objective optimization for green supply chain network design. Decision Support Systems, 51, 262-269. http://dx.doi. org/10.1016/j.dss.2010.11.020

Wu, Z., \& Pagell, M. (2011). Balancing priorities: Decisionmaking in sustainable supply chain management. Journal of Operations Management, 29, 577-590. http://dx.doi. org/10.1016/j.jom.2010.10.001

Wu, K., Tseng, M., \& Vy, T. (2011). Evaluation the drivers of green supply chain management practices in uncertainty. Procedia - Social and Behavioral Sciences, 25, 384-397. 
Ying, J., \& Li-Jun, Z. (2012). Study on Green Supply Chain Management Based on Circular Economy. Physics Procedia, 25, 1682-1688. http://dx.doi.org/10.1016/j. phpro.2012.03.295

Zhao, R., Neighbour, G., Han, J., Mcguire, M., Deutz, P. (2012). Using game theory to describe strategy selection for environmental risk and carbon emissions reduction in the green supply chain. Journal of Loss Prevention in the Process Industries, 25, 927-936. http://dx.doi. $\operatorname{org} / 10.1016 /$ j.jp. 2012.05.004

Zhu, Q., \& Dou, Y. (2007). Evolutionary Game Model between Governments and Core Enterprises in Greening Supply Chains. Systems Engineering - Theory \& Practice, 27(12) .

Zhu, Q., \& Sarkis, J. (2004a). The Link between Quality Management and Environmental Management in Firms of Differing Size: An Analysis of Organizations in China. Environmental Quality Management, 13(3), 53-64. http://dx.doi.org/10.1002/tqem.20004

Zhu, Q., \& Sarkis, J. (2004b). Relationships between operational practices and performance among early adopters of green supply chain management practices in Chinese manufacturing enterprises. Journal of Operations Management, 22, 265-289. http://dx.doi.org/10.1016/j. jom.2004.01.005

Zhu, Q., \& Sarkis, J. (2006). An inter-sectoral comparison of green supply chain management in China: Drivers and practices. Journal of Cleaner Production, 14, 472-486. http://dx.doi.org/10.1016/j.jclepro.2005.01.003
Zhu, Q., \& Sarkis, J. (2007). The moderating effects of institutional pressures on emergent green supply chain practices and performance. International Journal of Production Research, 45(18-19), 4333-4355. http:// dx.doi.org/10.1080/00207540701440345

Zhu, Q., Sarkis, J., \& Lai, K. (2007). Initiatives and outcomes of green supply chain management implementation by Chinese manufacturers. Journal of Environmental Management, 85, 179-189. PMid:17084502. http:// dx.doi.org/10.1016/j.jenvman.2006.09.003

Zhu, Q., Sarkis, J., \& Lai, K. (2008a). Green supply chain management implications for "closing the loop". Transportation Research Part E, 44, 1-18. http://dx.doi. org/10.1016/j.tre.2006.06.003

Zhu, Q., Sarkis, J., \& Lai, K. (2012). Green supply chain management innovation diffusion and its relationship to organizational improvement: An ecological modernization perspective. Journal of Engineering and Technology Management, 29, 168-185. http://dx.doi. org/10.1016/j.jengtecman.2011.09.012

Zhu, Q., Sarkis, J., Lai, K., \& Geng, Y. (2008b). The Role of Organizational Size in the Adoption of Green Supply Chain Management Practices in China. Corporate Social Responsibility and Environmental Management, 15, 322337. http://dx.doi.org/10.1002/csr.173

Zhu, Q., Geng, Y., Sarkis, J., \& Lai, K. (2011). Evaluating green supply chain management among Chinese manufacturers from the ecological modernization perspective. Transportation Research Part E, 47, 808821. http://dx.doi.org/10.1016/j.tre.2010.09.013

\title{
Green Supply Chain Management: a review of recent scientific production (2001-2012)
}

\begin{abstract}
The goal of this study was to analyze international scientific production in the area of constant-production green supply chain management from 2001 to 2012 using the Business Source Complete database (EBSCO Host). The database was checked for cooperation between authors and institutions, author entrants, production and continuity categories, regularity of publication and distribution of publications over time. Ninety articles were included in the sample, and the results showed a reduction in the number of publishing authors, concentrated in one-timers category with 68.90\%. The highest yield for a single author was 10 articles, and the most prolific periodical was the Journal of Cleaner Production, with 12 articles published on the subject. Clark University (USA) stood out in terms of output, with 12 affiliated authors. It was concluded that the subject had experienced a significant rise in published literature over that time period.
\end{abstract}

\section{Keywords}

Green Supply Chain Management. Management of green supply chains. Bibliometrics. Categories of production and continuity. 